\title{
DETERMINAÇÃO DE FÓSFORO EM PALEOSSEDIMENTOS EMPREGANDO ESPECTROMETRIA DE FLUORESCÊNCIA DE RAIOS-X PORTÁTIL
}

\author{
Jacira I. de Moura ${ }^{a, d}$, Benedito B. Farias Filho ${ }^{\mathrm{a}, *(1)}$, Maria Conceição S. M. Lage ${ }^{\mathrm{b}}$, Christian Rilza S. de Melo ${ }^{\mathrm{a}}$, Wilkins O. \\ de Barros ${ }^{a}$, Aníbal Gustavo S. Oliveira ${ }^{a}$ e Luzia Maria S. Carvalho ${ }^{c}$ \\ aDepartamento de Química, Universidade Federal do Piauí, 64049-550 Teresina - PI, Brasil

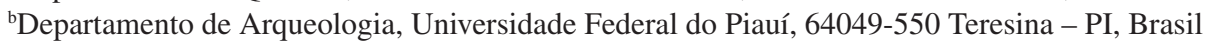

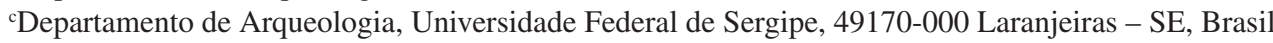 \\ ${ }^{\mathrm{d} I n s t i t u t o}$ Federal de Educação Ciência e Tecnologia do Maranhão, Campus Timon, 65635-468 Timon - MA, Brasil
}

Recebido em 09/05/2020; aceito em 04/08/2020; publicado na web em 10/09/2020

\begin{abstract}
DETERMINATION OF PHOSPHORUS IN PALAEOSEDIMENTS USING PORTABLE X-RAY FLUORESCENCE SPECTROMETRY. Phosphorus $(\mathrm{P})$ is an important chemical marker of the past human presence, because, besides indicates the localization of possible archaeological sites, also, contributes to clarify doubts regarding to origin, duration and to specific functions that were developed in them. The present study aimed to obtain a simple, accurate, fast and non-destructible analytical method to determine phosphorus in sediment samples of the Toca da Baixa dos Caboclos archaeological site, an enclave of the Serra da Capivara National Park, Piauí, Brazil. For the proposed method, a portable energy dispersive X-ray fluorescence ( $p$-ED-XRF) equipment and cross calibration to sample direct analysis were used. The results show that the concentrations obtained by the proposed method, cross calibration with use of $p$-ED-XRF, and by the spectrophotometric method were considered statistically equal, when compared by the paired $t$ test $(\mathrm{p}=0.05)$. By its turn, the intermediate accuracy and recovery tests showed values in the range of $2.41-3.56 \%$ and $85.10-102.66 \%$, respectively.
\end{abstract}

Keywords: phosphorus; palaeosediments; portable X-ray fluorescence.

\section{INTRODUÇÃO}

O estudo arqueométrico dos paleossedimentos pode ajudar a explicar a baixa presença de vestígios de natureza orgânica e fornecer, dentre outros dados, importantes marcadores de ocupação humana, essenciais ao processo de reconstrução de paleoambientes. ${ }^{1} \mathrm{O}$ fósforo (P), por exemplo, é um marcador químico que desperta grande interesse arqueológico há muito tempo, visto que é o único indicador, entre os elementos estudados, extremamente sensível e persistente à presença humana pretérita em locais onde há pouca ou nenhuma evidência superficial. ${ }^{2} \mathrm{~A}$ determinação dos níveis de fósforo no solo permite detectar não apenas traços da ocupação humana pretérita, mas sobretudo mudanças provocadas nas assinaturas físico-químicas do solo, além de apresentar um elevado potencial para informar sobre localização, origem, tipo, duração, intensidade e funções específicas associadas às atividades que eram desenvolvidas no local onde hoje se denomina de sítio arqueológico. ${ }^{3-6}$

A presença do fósforo no solo, de forma não natural, é originada por adições antropogênicas provenientes de fontes como: restos de animais e plantas, enterros, subprodutos metabólicos, queimadas, fertilizantes químicos no solo, entre outros. ${ }^{2,4,7,8}$ Assim, quando há o rompimento do homem com o ciclo desse elemento químico, ele se torna um indicador das ações humanas sobre o meio. E seu estudo, como marcador, parte sempre da premissa básica de que a deposição superficial da matéria orgânica, que contém fósforo orgânico, é proporcional ao período de ocupação humana, apresentando, portanto, níveis mais elevados que o natural no solo. ${ }^{1,2,9,10}$

Quando depositado no solo, como matéria orgânica ou inorgânica, o fósforo rapidamente se liga aos elementos químicos como $\mathrm{Al}$, $\mathrm{Ca}$ e $\mathrm{Fe}$ e, dependendo do $\mathrm{pH}$ e da atividade microbiana do solo, forma fosfatos minerais e ésteres de fosfato orgânico, relativamente

\footnotetext{
*e-mail: beneditofarias@ufpi.edu.br
}

estáveis. ${ }^{4,11,12}$ Esses fosfatos apresentam uma certa imobilidade em solos, além de serem menos suscetíveis à lixiviação, oxidação, redução ou absorção de plantas do que a maioria dos outros elementos indicadores da atividade humana. ${ }^{13}$ É essa facilidade de deposição, bem como sua capacidade de persistir no tempo e de ser estudado numa ampla faixa de $\mathrm{pH}$, que o torna o melhor marcador de detecção e de interpretação do assentamento humano pré-colonial., ${ }^{2,10}$

O Estado do Piauí é um dos principais representantes da arqueologia brasileira, devido à rica diversidade de vestígios arqueológicos encontrados ao longo do seu território, principalmente na região do Parque Nacional Serra da Capivara. ${ }^{5,14,15}$ Um desses vestígios são os sedimentos arqueológicos, encontrados em muitos de seus sítios, como, por exemplo, na Toca da Baixa dos Caboclos (TBC), Figura 1, localizado no limite entre os municípios de São João do Piauí e Capitão Gervásio Oliveira, coordenadas UTM 821272 E e $9065428 \mathrm{~N}$, que se destaca, sob a ótica arqueológica, por possuir estruturas funerárias de tempos remotos. ${ }^{16}$ Esse sítio é considerado "enclave" do Parque Nacional Serra da Capivara, o qual constitui uma vasta unidade de conservação, conhecida mundialmente por possuir milhares de sítios com variadas particularidades e que mostram evidências da ocupação humana pretérita há mais de 90 mil anos. ${ }^{17}$

As transformações que ocorrem no solo, durante o período de deposição de um indicador químico, podem ser tão marcantes que apontam para uma configuração bem diferente da existente no momento da ocupação. Essas alterações podem ser avaliadas por meio da aplicação de métodos analíticos, que identifiquem as concentrações de elementos químicos indicadores da passagem humana em um local. ${ }^{2,6}$ Então, quando os sítios, a exemplo do TBC, possuem seus paleossedimentos analisados, os resultados obtidos podem ser aliados a estudos multi e interdisciplinares, ajudando, com isso, a traçar um esboço da pré-história regional, desde a chegada dos primeiros grupos humanos até os dias atuais. ${ }^{18}$ 


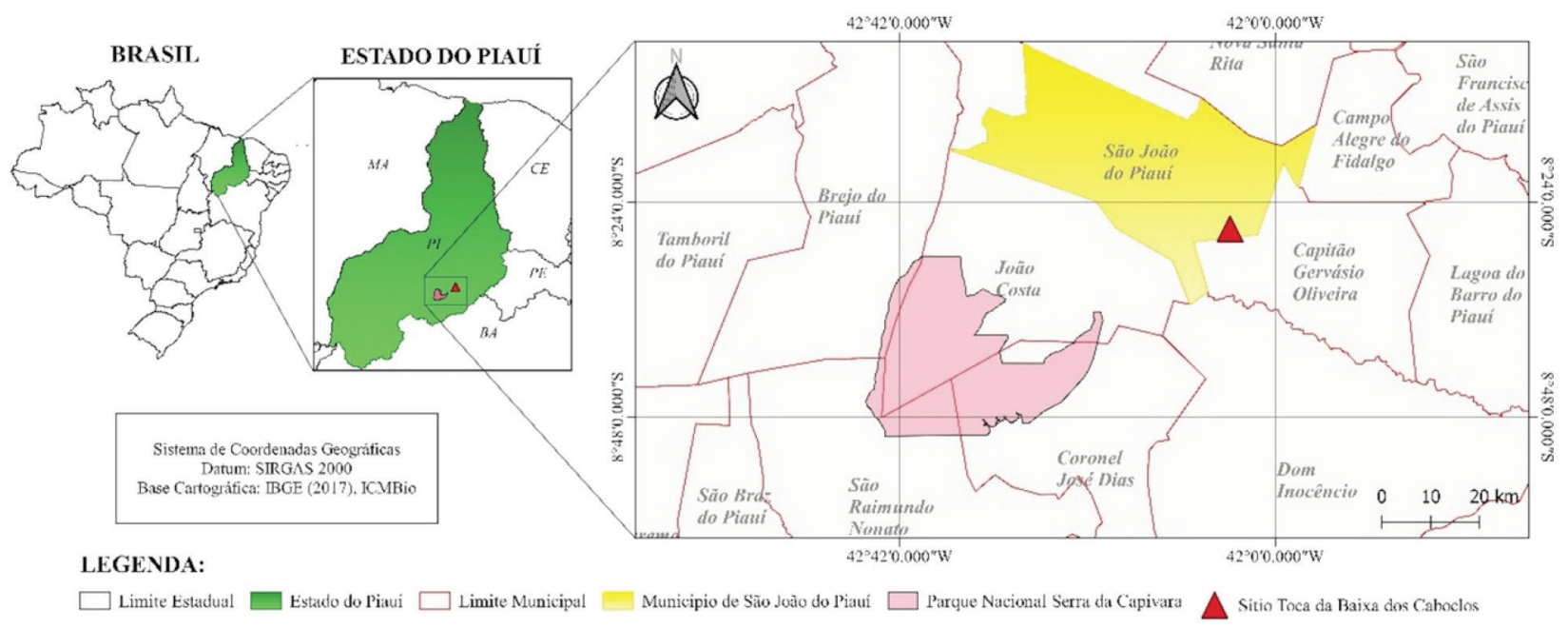

Figura 1. Localização geográfica do Parque Nacional Serra da Capivara e do Sítio Toca da Baixa dos Caboclos

A literatura científica reporta como metodologias base para extrair e medir fósforo, nas suas diversas formas (P disponível, $\mathrm{P}$ orgânico, $\mathrm{P}$ inorgânico e $\mathrm{P}$ total) em solos arqueológicos, aquelas que fazem uso, principalmente, de técnicas como: colorimetria ${ }^{1,19-23}$ e plasma indutivamente acoplado (ICP). ${ }^{24,25}$ A maioria dos métodos colorimétricos, incluindo o espectrofotométrico de absorção molecular na região do visível (Vis), que é amplamente utilizado na determinação de fósforo em sedimentos, tem por base o trabalho de Murphy e Rilley, ${ }^{26}$ a partir do qual foi formado o complexo azul de molibdênio. ${ }^{27,28}$ No entanto, esses métodos podem apresentar alguns inconvenientes como: uso de equipamentos caros, extensivo preparo da amostra e metodologias invasivas, tornando-se, em alguns casos, inviáveis.

Em oposição às técnicas citadas, Marwick ${ }^{3}$ apresentou em seu trabalho o uso da técnica de espectrometria de fluorescência de raios-X (XRF) para determinar baixos níveis de fósforo em amostras sedimentológicas. Essa técnica tem sido utilizada com sucesso no mundo todo para caracterização do solo, e os trabalhos que fazem uso dela para análises de sedimentos arqueológicos estão ganhando cada vez mais espaço nos últimos anos, principalmente após o surgimento dos equipamentos portáteis, pois, além de ser uma técnica de fácil execução, ainda viabiliza que as análises de amostras de sedimentos sejam realizadas sem a necessidade de solubilização delas, preservando-as e, também, evitando os problemas enfrentados pelos métodos de preparo de amostras via úmida. ${ }^{29-32}$ Mas, no Brasil, a utilização do espectrômetro de fluorescência de raios-X (ED-XRF) para estudo dos solos ainda é incipiente, sobretudo, com relação ao espectrômetro de fluorescência de raios-X portátil ( $p$-ED-XRF).$^{30}$

$\mathrm{O}$ uso do $p$-ED-XRF pode trazer inconvenientes de interferência espectral, que surgem quando os raios X característicos são coletados e medidos simultaneamente pelo detector. Se ocorrer, durante as medidas, a emissão de um fóton de $\mathrm{Si}(1,74 \mathrm{keV})$ proveniente do detector (pico de escape), cada pico do espectro associado à amostra terá sua energia inferior a $1,74 \mathrm{keV}$, ou seja, a linha $\mathrm{K} \alpha$ do cálcio $(3,69 \mathrm{keV})$ corresponderá no espectro à energia de $1,95 \mathrm{keV}$, o que poderia causar uma interferência espectral ao fósforo $(2,01 \mathrm{keV}) \mathrm{e}$ tornar as medidas para amostras sedimentológicas menos precisas, por exemplo. ${ }^{33}$ Como se trata de uma possível interferência espectral, causada por condições instrumentais, se ela ocorrer, poderá ser minimizada ou corrigida das seguintes maneiras: (1) selecionando o pico alternativo livre de interferência; (2) limitando a faixa de energia para medição do pico; (3) usando modelos matemáticos para a subtração da interferência ou (4) utilizando alternativas experimentais. ${ }^{34}$
Para Holliday e Gartner, ${ }^{2}$ os vários métodos desenvolvidos para determinação de fósforo no solo podem resultar numa volumosa quantidade de dados e interpretações, causando confusão sobre a não apropriação de métodos e terminologia. Por isso, são de grande relevância pesquisas que desenvolvam métodos analíticos mais céleres e confiáveis para determinação de fósforo em sedimentos arqueológicos. E, nesse caso, um método que faça uso da técnica de espectrometria de fluorescência de raios-X (XRF), com uso de equipamento portátil, pode surgir como alternativa aos métodos já desenvolvidos e trazer vantagens como simplicidade, baixo custo, não pré-tratamento da amostra, redução do número de etapas, informações espectrais relativamente simples com mínima interferência espectral, exatidão, precisão, capacidade de analisar amostras de pequenas dimensões, rapidez na análise, além de não intervenção, dependendo do método a ser aplicado. Nesse sentido, o objetivo deste trabalho foi desenvolver um método analítico utilizando a espectrometria de fluorescência de raios-X portátil ( $p$-XRF) para determinar fósforo em amostras de sedimentos arqueológicos.

\section{PARTE EXPERIMENTAL}

\section{Instrumentação}

Para o preparo de amostras e determinação quantitativa do fósforo, foram utilizados os seguintes equipamentos: espectrofotômetro de absorção molecular na região do visível (Bel Engineering, V-M5, Monza, Milano, Itália); espectrômetro portátil de fluorescência de raios X por dispersão de energia, $p$-ED-XRF (Thermo Fischer, Scientific Niton XL3t ultra, Waltham, Massachusetts, USA); balança analítica (Denver, APX-200, Arvada, Colorado, USA); mufla (EDG, 10 P-S, São Carlos, São Paulo, Brasil); mesa agitadora (Quimis, Q225M, Diadema, São Paulo, Brasil) e sistema Milli-Q de purificação de água (Elga, Purelab Option-Q, Woodridge, Illinois, USA).

\section{MATERIAIS E REAGENTES}

Para as determinações por método espectrofotométrico, foram utilizadas cubetas de vidro com $1 \mathrm{~cm}$ de caminho ótico e soluções padrão de: ácido ascórbico (Dinâmica, Indaiatuba, SP, Brasil) a 0,57 $\mathrm{mol} \mathrm{L}^{-1}$, ácido clorídrico (Qhemis, Jundiaí, SP, Brasil) a 1,00 mol L-1, ácido sulfúrico (Merck, Baurueri, SP, Brasil) a 4,50 $\mathrm{mol} \mathrm{L}^{-1}$, fosfato de potássio monobásico anidro (Synth, Diadema, SP, Brasil) a 100,00 mg L-1 , molibdato de amônio (Merck, Baurueri, 
SP, Brasil) a 0,02 $\mathrm{mol} \mathrm{L}^{-1}$ e tartarato de antimônio e potássio (Merck, Baurueri, SP, Brasil) a $0,06 \mathrm{~mol} \mathrm{~L}^{-1}$. Todas as soluções foram preparadas com água deionizada (resistividade de $18,2 \mathrm{M} \Omega \mathrm{cm}$ ) e reagentes de grau analítico. Na etapa das determinações por $p$-ED-XRF, fez-se uso dos reagentes fosfato de potássio monobásico anidro P. A. (Synth, Diadema, SP, Brasil) e cloreto de cálcio dihidratado P. A. (Dinâmica, Indaiatuba, SP, Brasil). Os materiais de laboratório e vidrarias foram previamente limpos para descontaminação por imersão em solução de ácido nítrico (Dinâmica, Indaiatuba, SP, Brasil) a $10 \% \mathrm{v} \mathrm{v}^{-1}$, por no mínimo 24 h.

\section{Amostras}

Foram estudadas onze amostras de sedimentos arqueológicos, depositadas na reserva técnica do Instituto Sérgio Motta/FUMDHAM (Fundação Museu do Homem Americano), localizado na cidade de São Raimundo Nonato-PI. Elas são oriundas do Sítio Arqueológico Toca da Baixa dos Caboclos, enclave do Parque Nacional Serra da Capivara, e foram coletadas durante etapas de escavação, que ocorreram entre os meses de janeiro a março de 1998, por uma equipe interdisciplinar da FUMDHAM. As amostras (Tabela 1) foram selecionadas de níveis estratigráficos pertencentes a diferentes contextos funerários (enterramentos) e a quantidade delas, retirada de cada enterramento, teve por base os teores de fósforo previamente detectados por espectrofotometria. A amostra branco do sedimento - livre de fósforo antrópico e com baixa concentração de fósforo natural - foi coletada em um local extra sítio.

Em laboratório, as amostras foram devidamente catalogadas, homogeneizadas, peneiradas em malha de nylon com diâmetro de $75 \mu \mathrm{m}$, secas em temperatura ambiente e estocadas em frascos de polipropileno. Em seguida, foram separadas oito amostras de sedimentos para a construção da curva analítica (amostras de calibração) e três amostras externas à calibração, usadas para prever a sua concentração.

Tabela 1. Descrição dos paleossedimentos analisados

\begin{tabular}{ccl}
\hline Amostras & Localização & \multicolumn{1}{c}{ Descrição } \\
\hline Branco analítico & & Sedimento coletado extra sítio \\
\hline E01-SD01 & Enterramento 01 & $\begin{array}{l}\text { Urna de uma criança do sexo } \\
\text { masculino }\end{array}$ \\
\hline E01-SD02 & Enterramento 03 & Urna de uma criança \\
\hline E03-SD01 & Enterramento 06 & $\begin{array}{l}\text { Urna de um adulto do sexo } \\
\text { masculino }\end{array}$ \\
\hline E06-SD01 & Enterramento 07 & $\begin{array}{l}\text { Urna de um adulto do sexo } \\
\text { anos de idade }\end{array}$ \\
\hline E07-SD01 & & Urna de uma criança \\
E07-SD02 & Enterramento 08 & $\begin{array}{l}\text { Urna de uma criança lactante de } \\
\text { cerca de 6 meses de idade }\end{array}$ \\
\hline E07-SD03 & Enterramento 09-SD01
\end{tabular}

\section{Procedimento analítico}

\section{Determinação de fósforo por método espectrofotométrico}

O método utilizado baseia-se no trabalho de Cavalcante, ${ }^{1}$ que adaptou o método de Murphy e Rilley ${ }^{26}$ para determinação de fósforo em águas naturais à determinação de fósforo em sedimentos. $\mathrm{O}$ estudo consiste em adicionar à amostra uma solução acidificada de molibdato de amônio, contendo ácido ascórbico e uma pequena quantidade de antimônio, o que ocasiona a formação e a redução do ácido fosfomolibdico. O máximo de absorção do complexo azul de fosfomolibdênio na presença de antimônio ocorre em $\sim 880 \mathrm{~nm}$.

Para obtenção da concentração de fósforo total, uma massa de $0,1000 \mathrm{~g}$ de cada amostra de sedimento arqueológico foi calcinada a $550{ }^{\circ} \mathrm{C}$ durante $1 \mathrm{~h}$. Após resfriamento, o resíduo recebeu o acréscimo de 25,00 mL de solução de ácido clorídrico $1,0 \mathrm{~mol} \mathrm{~L}^{-1} \mathrm{e}$ foi submetido à agitação mecânica por 16 h a $170 \mathrm{rpm}$. Em seguida, as amostras foram filtradas, e, de cada filtrado obtido, retirou-se uma alíquota de $2 \mathrm{~mL}$ para que fosse realizado o processo de formação do complexo azul de fosfomolibdênio. A determinação das concentrações de fósforo nas amostras foi feita por interpolação das absorbâncias na curva de calibração, com faixa de trabalho entre 0,10 e $1,60 \mathrm{mg} \mathrm{L}^{-1}$ de fósforo.

\section{Determinação de fósforo por $p-E D-X R F$}

$\mathrm{O}$ espectrômetro de $p$-ED-XRF utilizado possui um tubo de raios-X (com anodo de prata), um detector de deriva de silício (SDD), voltagem máxima de $50 \mathrm{kV}$, corrente de $200 \mu \mathrm{A}$ e potência de $2 \mathrm{~W}$. O equipamento possui quatro filtros denominados: main, low, high $\mathrm{e}$ light, e cada um deles opera de forma sensível para um conjunto de elementos químicos. Foi selecionado o filtro light, visto que possui sensibilidade maior para o fósforo, elemento em estudo. Todas as amostras foram analisadas de forma direta, dispensando a etapa de abertura da amostra.

Escolheu-se uma das amostras de sedimento (E01-SD01) para ajuste de parâmetros analíticos e instrumentais. A amostra em estudo foi colocada em um colimador de $32 \mathrm{~mm}$ com filme de polipropileno de $4 \mu \mathrm{m}$ de espessura e, depois, analisada sob atmosfera. Depois, foram realizadas medidas para avaliar o sinal analítico do fósforo $(2,014 \mathrm{keV})$ na amostra, de acordo com: a massa utilizada $(0,5 ; 1,0$ e $1,5 \mathrm{~g}$ ), a granulometria (amostra bruta ou peneirada em malha de $75 \mu \mathrm{m})$, o tempo de análise $(30,60,90,100,120$ e 150 s) e o spot (área de irradiação de 7 e $50 \mathrm{~mm}^{2}$ ), função disponível no equipamento. Também foi realizado um ensaio para verificar a variação em torno do sinal analítico obtido. Todas as medidas foram realizadas em triplicata e em diferentes pontos das amostras.

Após o estudo dos parâmetros citados, realizaram-se novas medidas para verificar se o detector de deriva de silício do $p$-ED-XRF geraria picos de escape, fazendo com que o cálcio, presente em sedimentos arqueológicos, apresentasse sinal analítico em 1,95 $\mathrm{keV}$, interferindo, dessa maneira, na linha espectral do fósforo em 2,014 keV. Para tanto, foram realizadas medidas espectrométricas por $p$-ED-XRF nos reagentes químicos fosfato de potássio monobásico anidro e cloreto de cálcio dihidratado - em razão de possuírem, respectivamente, o analito de interesse e o possível interferente - usando-se os parâmetros já definidos para as determinações de fósforo. Também foram realizadas medidas na amostra de sedimento E01-SD01 mediante ativação apenas do filtro light, assim como dos filtros low (de maior sensibilidade para cálcio) e light, simultaneamente, durante as medidas.

\section{Calibração cruzada}

Para a realização da análise direta de sedimento para determinação de fósforo empregando $p$-ED-XRF, preparou-se uma calibração externa utilizando como padrões oito das 11 amostras sedimentológicas disponíveis, escolhidas de forma aleatória. Assim, a curva analítica para determinação de fósforo nas amostras foi obtida plotandose as intensidades dos sinais de emissão lidas pelo equipamento $p$-ED-XRF versus as massas das amostras $\left(\mathrm{mg} \mathrm{g}^{-1}\right)$ obtidas por medida espectrofotométrica na região do Visível. As concentrações de fósforo, no conjunto das três amostras externas à curva analítica, foram determinadas por calibração cruzada por meio da interpolação das intensidades de emissão obtidas por $p$-ED-XRF. 
Após a determinação da concentração de fósforo nas amostras externas à curva analítica, foram avaliados parâmetros analíticos como: limite de detecção (LD), limite de quantificação (LQ), repetitividade, precisão intermediária e exatidão do método proposto. Para os ensaios de precisão e exatidão, as amostras possuíam três níveis diferentes de concentração. A exatidão das medidas foi avaliada por meio do método de adição e recuperação de analito. Para o método, utilizaram-se 9 determinações, em 3 níveis de concentrações, com 3 réplicas em cada nível. Depois da adição do analito, as amostras foram homogeneizadas antes de se iniciar as medidas.

\section{RESULTADOS E DISCUSSÃO}

As determinações analíticas do teor de fósforo nas amostras de sedimentos foram realizadas após seleção do método de calibração do equipamento denominado de mining $\mathrm{Cu} / \mathrm{Zn}$, fazendo-se uso do filtro light, devido à sua maior sensibilidade ao elemento químico de interesse. A Figura 2 apresenta o resultado da análise qualitativa de um dos sedimentos (E01-SD01), destacando a linha espectral do $\mathrm{P}_{\mathrm{k} \alpha}$ em 2,014 keV, a qual foi selecionada para a realização das medidas quantitativas e para otimização dos parâmetros analíticos e instrumentais, em virtude de sua maior intensidade de sinal. Vale a pena destacar que foi eleita apenas uma das amostras analisadas para a representação do perfil espectral (Figura 2), uma vez que as demais foram bastante similares.

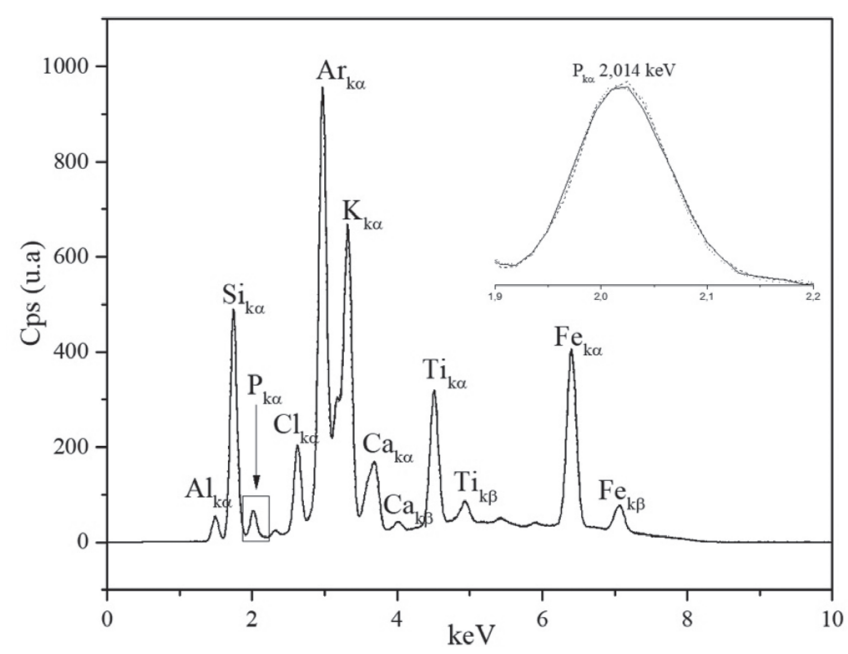

Figura 2. Espectros de Fluorescência de Raios X resultantes de três medidas analíticas da amostra do paleossedimento E01-SD01, com destaque para a linha atômica $k_{\alpha}$ do fósforo em $2,014 \mathrm{keV}$

Para a atribuição elementar das linhas atômicas (Figura 2), foram consideradas as energias associadas às linhas $\mathrm{K} \alpha$ e $\mathrm{K} \beta$, características de cada elemento, e a intensidade dos sinais analíticos. Como pode ser observado, além do fósforo foram detectados outros elementos, como $\mathrm{Si}$ e $\mathrm{Al}$, oriundos de matriz proveniente de aluminossilicatos em suas diversas formas, que inclui em sua estrutura elementos como $\mathrm{Fe}, \mathrm{K}$, $\mathrm{Ca}, \mathrm{Ti}$. A elevada intensidade analítica do elemento Ferro pode estar associada às espécies químicas na forma de óxido, presentes em solos hematíticos $\left(\mathrm{Fe}_{2} \mathrm{O}_{3}\right)$. O cloro detectado nas análises pode ser derivado do intemperismo da rocha, considerando que se trata de um ambiente em que se encontram sais de eflorescência salina nos paredões rupestres. Por fim, o pico de alta intensidade observado para o argônio $(\mathrm{K} \alpha=2,957$ $\mathrm{keV}$ ) pode ser explicado pela presença desse elemento no ar ambiente. ${ }^{35}$

A espessura da amostra estudada, aqui relacionada com a massa da amostra acomodada no colimador, pode produzir um efeito significativo sobre o sinal analítico obtido, devido à fluorescência emitida pelo fósforo, que pode ser absorvida pelas camadas mais profundas do paleossedimento. ${ }^{36}$ Dessa forma, faz-se necessário conhecer a massa ideal a ser analisada na qual o efeito da matriz sobre a análise seja mínimo, ou por meio da qual se possa alcançar uma sensibilidade adequada. A variação do sinal analítico do fósforo (Figura 3A) em função da massa de amostra estudada mostrou que houve um ganho significativo de sinal (cerca de 50\%) com 1,0 g. Após esse valor, o sinal analítico obtido parece não alterar muito e, portanto, para evitar uso excessivo de amostra, foi selecionado esse valor em massa para realização das medidas por $p$-ED-XRF.

O comportamento analítico, considerando o parâmeto granulometria (Figura 1S, material suplementar), revela que o tamanho de partícula interfere, consideravelmente, no ganho de sinal analítico do fósforo, posto que a amostra bruta apresentou menor intensidade quando comparada à amostra peneirada, realizando a análise no intervalo de tempo de $90 \mathrm{~s}$ e sem uso do recurso instrumental spot. Esse resultado se deve à heterogeneidade das partículas, uma vez que diferentes tamanhos dos grãos dos paleossedimentos podem apresentar variáveis sítios de sorção do fósforo, causando flutuações nas medidas analíticas. ${ }^{37} \mathrm{~A}$ fração mais fina dos paleossedimentos - a argilosa - apresenta uma área superficial muito maior para a sorção de metais em comparação com frações de tamanho maior, isto é, sem passar por qualquer etapa de peneiração. ${ }^{38,39}$ Dessa forma, a United States Environmental Protection Agency (USEPA) ${ }^{40}$ recomenda o uso de frações granulométricas de $<250 \mu \mathrm{m}$, em conformidade com a utilizada neste trabalho $(75 \mu \mathrm{m})$. Logo, em atividades in situ há a necessidade da etapa de peneiração para obtenção das frações mais finas dos paleossedimentos a serem utilizadas para as medidas analíticas por $p$-ED-XRF.

Alguns equipamentos de $p$-ED-XRF oferecem aos analistas a opção de variar o tamanho do feixe (spot) que é incidido sobre a amostra, a fim de selecionar o melhor sinal obtido, considerando sua heterogeneidade. Assim, é importante realizar várias medições usando diferentes diâmetros de feixe $(3$ e $8 \mathrm{~mm}$ ) de incidência de Raios X para verificar a influência desta ferramenta sobre o sinal analítico obtido. ${ }^{41}$ A Figura $3 \mathrm{~B}$ apresenta o resultado do estudo realizado com o uso do recurso spot (áreas de irradiação do feixe de $7 \mathrm{~mm}^{2}$ ) e sem o uso desse recurso (áreas de irradiação do feixe de $50 \mathrm{~mm}^{2}$ ), em triplicata, no intervalo de tempo de $90 \mathrm{~s}$. Nota-se que a ausência do recurso spot se torna mais viável para as análises em estudo, em razão de haver um ganho de sinal analítico maior que o dobro. Ao contrário, a utilização de uma área de feixe reduzida para $7 \mathrm{~mm}^{2}$ diminui consideravelmente o sinal obtido, em decorrência da pequena área analisada onde o fósforo se encontra na amostra.

A Figura 3C apresenta os resultados obtidos em relação à intensidade da linha atômica do fósforo, de acordo com a variante tempo e sem o uso do spot. Em análise quantitativa, usando espectrometria de fluorescência de raios X, a variável tempo de irradiação é importante quando se deseja obter resultados mais precisos e de melhor sensibilidade, pois, de acordo com Kalnicky e Singhvi ${ }^{42}$ e Parsons et al., ${ }^{43}$ é importante otimizar os tempos de varredura, determinando a condição ideal para cada tipo de amostra, uma vez que este parâmetro pode variar de acordo com a configuração do instrumento, do analito de interesse e da matriz. Tighe e $_{\text {al. }}{ }^{44}$ também afirmaram que um aumento no tempo de análise permite reduzir os limites de detecção e manter boa relação sinal/ruído. Medidas realizadas em menor espaço de tempo de irradiação estão sujeitas a desvios maiores, em virtude de o tempo de contagem ser menor e, em amostras heterogêneas como sedimentos, a relação sinal/ruído ficaria substancialmente afetada, fato observado para os tempos de 30 e 60 segundos. No entanto, o uso de um maior tempo maior para medida pode favorecer o espalhamento da radiação, ocasionando um pronunciado efeito no desvio das determinações, também observado em 120 e 150 segundos.

Os resultados apresentaram um ganho de sinal analítico até o 

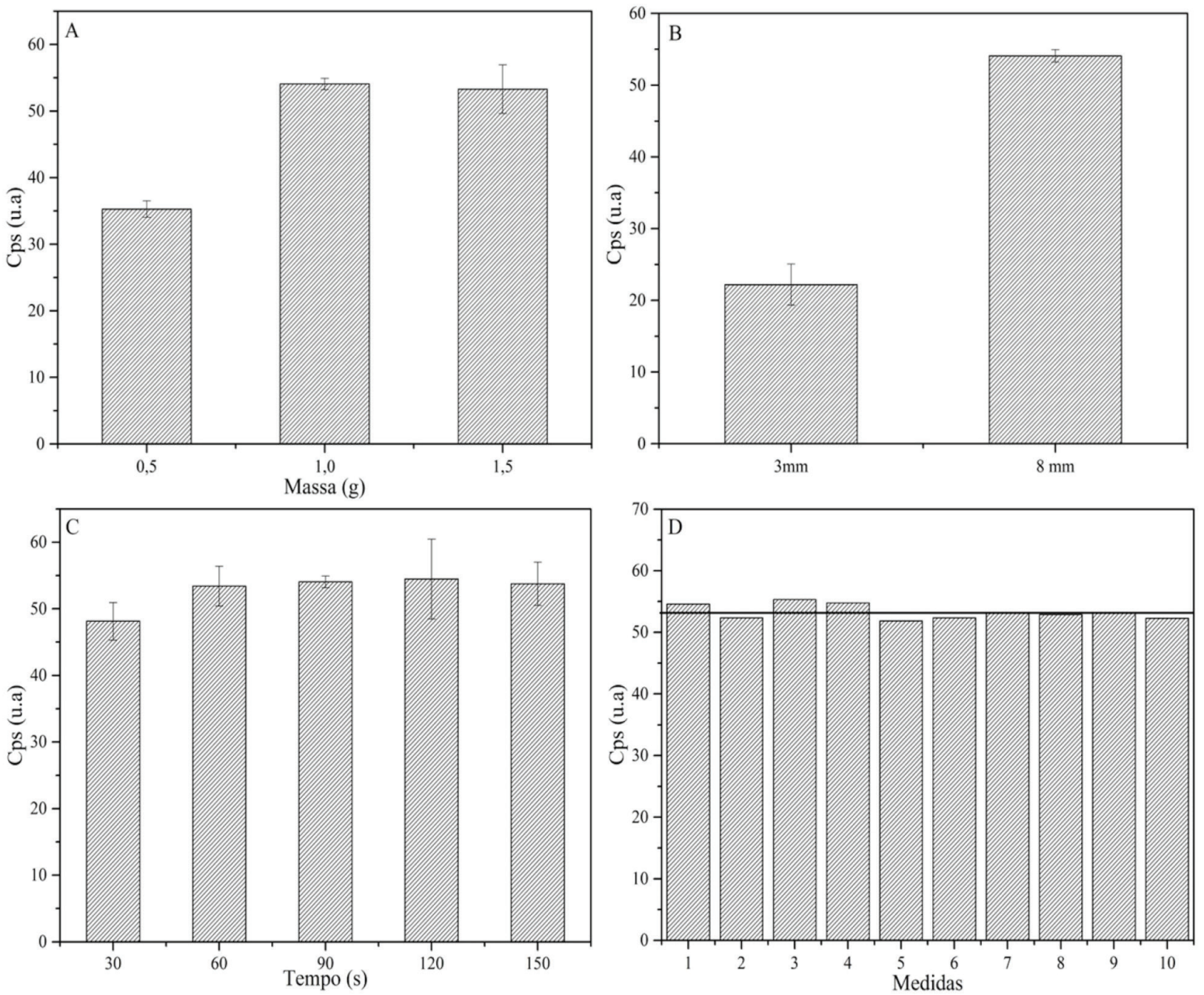

Figura 3. Intensidade média do sinal analítico do fósforo em 2,014 keV, do paleossedimento E01-SD01, resultante de três medidas analíticas para: (A) a massa da amostra utilizada na análise, (B) o diâmetro do feixe de incidência de Raios $X$ (3 e $8 \mathrm{~mm}),(C)$ o tempo de medida e (D) a variação em torno das intensidades dos sinais analíticos do fósforo para 10 medidas realizadas a cada 10 minutos

tempo de estudo de $90 \mathrm{~s}$ e a partir desse tempo não houve acréscimo na intensidade da linha atômica do fósforo, frente aos outros tempos estudados. Resultados semelhantes, em que os sinais analíticos das medidas realizadas por $p$-ED-XRF melhoraram, também foram obtidos por Kilbride et al. ${ }^{45}$ com o aumento do tempo de varredura até um tempo ótimo de análise, e a partir dele não houve mais ganho de sinal significativo. Ressalta-se, ainda, que foi nesse tempo de análise que se encontrou o menor desvio padrão e no qual os efeitos oriundos de pequenos tempos de contagem e de espalhamento foram mínimos. Assim, para tornar o procedimento analítico ainda mais célere, optou-se por analisar todas as amostras de paleossedimentos no intervalo de tempo de $90 \mathrm{~s}$, uma vez que ele apresentou maior ganho significativo de sinal analítico e menor desvio no valor das medidas.

Os espectros obtidos a partir das medidas realizadas por $p$-ED-XRF nos reagentes químicos fosfato de potássio monobásico anidro e cloreto de cálcio dihidratado, bem como no sedimento E01-SD01, estão apresentados na Figura 2S (Material Suplementar). Eles revelaram que, para os citados reagentes, o detector de deriva de silício do $p$-ED-XRF não gerou picos de escape, devido à não observação de uma linha espectral para o cálcio em 1,95 keV. Portanto, não houve interferência na linha espectral do fósforo em 2,014 keV. Em relação às medidas realizadas no sedimento E01-SD01, também não foi observada interferência espectral do cálcio sobre a linha $\mathrm{K} \alpha$ do fósforo quando ativado apenas o filtro light ou este juntamente com o filtro low. A intensidade observada na linha 2,014 keV a partir do momento em que se ativou o filtro low é característica do fósforo, pois, apesar de não ser o filtro de maior sensibilidade para este elemento químico, sua presença na amostra pode ser apontada pelos quatro filtros (main, low, high e light) presentes no equipamento, ainda que em baixa intensidade. A Figura 3D apresenta o resultado da variação em torno do sinal analítico fornecido pelo equipamento. Foram realizadas 10 medições, com intervalos de tempo de 10 minutos entre elas, e o desvio padrão relativo obtido a partir das medidas foi $2,26 \%$, apontando, assim, para valores concordantes entre si.

A Figura 4 apresenta a comparação dos valores de fósforo obtidos pela calibração interna do $p$-ED-XRF com os valores obtidos por espectrofotometria de absorção molecular. Para todas as amostras de sedimentos, as concentrações obtidas por $p$-ED-XRF apresentaram valores acima daqueles alcançados pelo método espectrofotométrico, assim, como não houve semelhança entre os valores conseguidos pelos dois métodos, acredita-se que a complexa composição química da matriz sedimentológica pode ter interferido nos valores dos sinais analíticos obtidos por sua calibração interna. Apesar de trabalharem com uma matriz diferente, Herreros-Chavez et al. ${ }^{46}$ observaram esse mesmo comportamento e também atribuíram essa diferença ao fato de a matriz não ter se ajustado ao método de calibração usado para quantificação do analito. Brito, Teixeira e Korn ${ }^{47}$ também observaram os efeitos gerados pela matriz em análises realizadas por ED-XRF. 


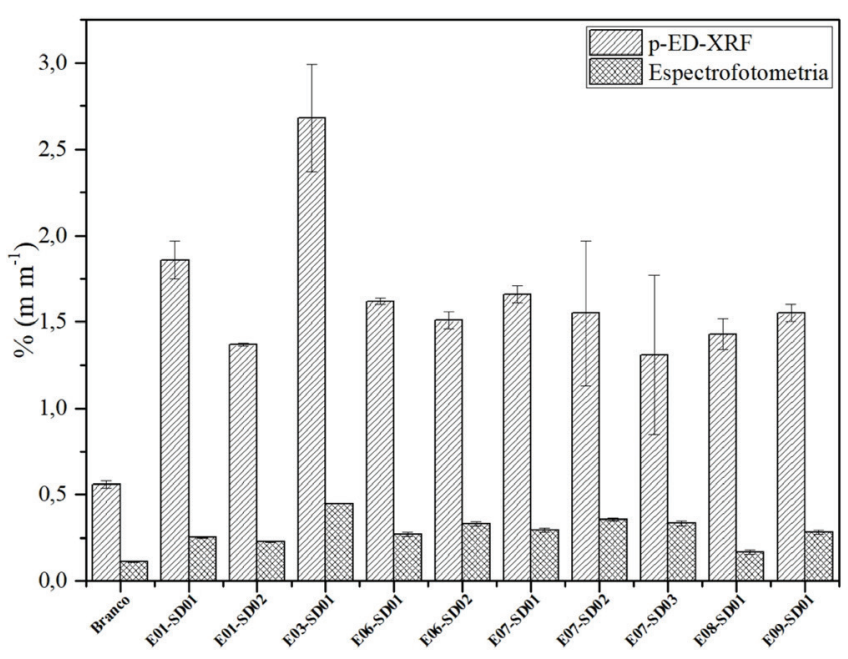

Figura 4. Comparação entre as concentrações de fósforo obtidas por --ED-XRF e espectrofotometria de absorção molecular em amostras de paleossedimentos

Para suavizar ou mesmo neutralizar o efeito da matriz, realizou-se uma calibração externa (Figura 5) empregando oito das 11 amostras disponíveis, em que as intensidades dos sinais obtidos por $p$-ED-XRF estão relacionadas com as concentrações do analito (em $\mathrm{mg} \mathrm{P} \mathrm{g}^{-1}$ de sedimento), conseguidas por espectrofotometria de absorção molecular, apontando para uma boa relação linear $(r=0,9477)$ dentro da faixa de concentração estudada. Os parâmetros analíticos para análise do fósforo estão expressos na Tabela 2. Os limites de detecção (LD) e de quantificação (LQ) foram calculados usando equações apresentadas no trabalho de Costa et al. ${ }^{48}$

Realizou-se a interpolação das três amostras externas à curva

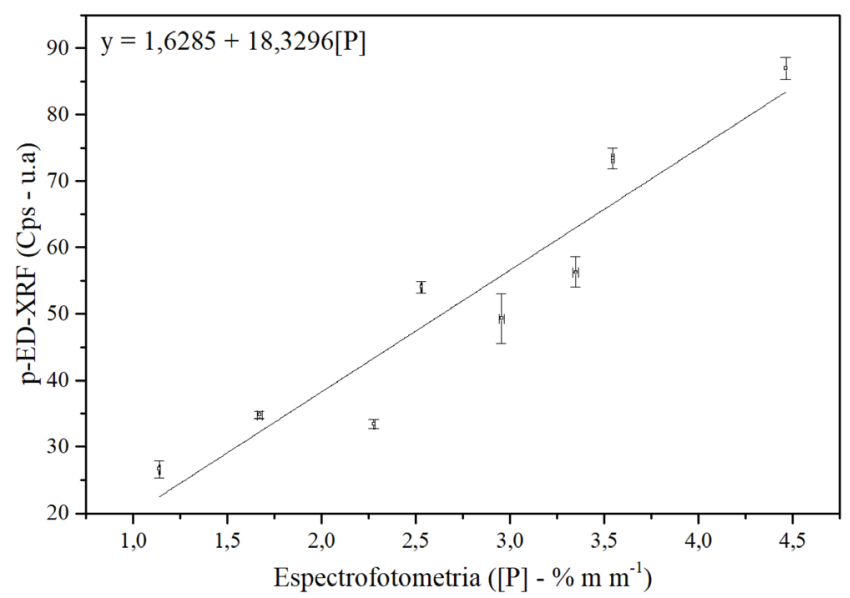

Figura 5. Curva analítica obtida pelas intensidades dos sinais de fósforo por p-ED-XRF, em 2,014 keV, versus suas, respectivas, concentrações por espectrofotometria de absorção molecular

Tabela 2. Parâmetros analíticos para determinação de fósforo em sedimentos arqueológicos por calibração cruzada com espectrofotometria de absorção molecular versus $p$-ED-XRF

\begin{tabular}{lc}
\hline Parâmetros analíticos & \\
\hline Coeficiente de correlação Linear $(\mathrm{r})$ & 0,9477 \\
Faixa de trabalho $\left(\mathrm{mg} \mathrm{g}^{-1}\right)$ & $1,14-4,46$ \\
LD $\left(\mathrm{mg} \mathrm{g}^{-1}\right)$ & 0,09 \\
LQ $\left(\mathrm{mg} \mathrm{g}^{-1}\right)$ & 0,28 \\
\hline
\end{tabular}

analítica a fim verificar se há concordância entre as concentrações de fósforo, obtidas por calibração cruzada com $p$-ED-XRF e espectrofotometria de absorção molecular. As concentrações alcançadas pelos dois métodos (Tabela 3 ) foram comparados por teste $t$ pareado $(\mathrm{p}=0,05)$, sendo consideradas estatisticamente iguais $\left(\mathrm{t}_{\mathrm{cal}} 3,95<\mathrm{t}_{\mathrm{tab}} 4,30\right)$.

Tabela 3. Concentrações de fósforo obtidas por calibração cruzada com $p$-ED-XRF e espectrofotometria de absorção molecular

\begin{tabular}{ccc}
\hline Amostras & $\begin{array}{c}\text { Espectrofotometria } \\
\left(\mathrm{mg} \mathrm{g}^{-1}\right)\end{array}$ & $\begin{array}{c}\text { Calibração Cruzada } \\
\left(\mathrm{mg} \mathrm{g}^{-1}\right)\end{array}$ \\
\hline E06-SD01 & 2,69 & 2,21 \\
E06-SD02 & 3,31 & 2,28 \\
E09-SD01 & 2,83 & 2,24 \\
\hline
\end{tabular}

Foram realizados ensaios de precisão intra-dia e intermediária, utilizando-se uma das três amostras (E09-SD01) externas à curva, em três níveis de massa: $0,500 \mathrm{~g}, 0,7500 \mathrm{~g}$ e $1,000 \mathrm{~g}$. Os resultados dos ensaios realizados, em triplicata, estão expressos na Tabela 4 e revelam que o método estudado apresenta boa precisão intermediária, com desvios padrão relativos variando entre 2,41 e $3,55 \%$. Ao comparar as precisões intra-dia e intermediária pelo teste $F$, observouse que, para os três níveis de concentração estudados, o valor de $F$ calculado $(2,67)$ é menor que o valor tabelado $(19,00)$, não havendo, assim, diferença significativa entre os resultados a $95 \%$ de confiança.

Tabela 4. Resultados do ensaio de precisão intra-dia e intermediária, utilizando-se calibração cruzada com $p$-ED-XRF versus espectrofotometria de absorção molecular

\begin{tabular}{ccc}
\hline & \multicolumn{2}{c}{ DPR (\%) } \\
\cline { 2 - 3 } Massa analisada (g) & $\begin{array}{c}\text { Precisão intra-dia (Ana- } \\
\text { lista 1) }\end{array}$ & $\begin{array}{c}\text { Precisão intermediária } \\
\text { (Analista 2) }\end{array}$ \\
\hline $0,5000 \mathrm{~g}$ & 2,42 & 2,66 \\
$0,7500 \mathrm{~g}$ & 3,77 & 2,41 \\
$1,0000 \mathrm{~g}$ & 1,85 & 3,56 \\
\hline
\end{tabular}

DPR- desvio padrão relativo.

Realizou-se, ainda, um teste de adição e recuperação de analito (Tabela 5) para três níveis de concentração de fósforo. A amostra E09-SD01 foi a escolhida para o ensaio, uma vez que ela possuía uma maior quantidade, em massa, quando comparada às outras amostras. Os ensaios foram realizados com 1,0000 $\mathrm{g}$ de sedimento e pequenas quantidades, em massa, de analito, para que o sinal analítico obtido após sua adição à amostra ficasse na faixa de interpolação da curva analítica.

Tabela 5. Resultados do ensaio de recuperação utilizando calibração cruzada p-ED-XRF versus espectrofotometria de absorção molecular

\begin{tabular}{lccc}
\hline Amostra & $\begin{array}{c}\text { Massa de } \mathrm{P} \\
\text { adicionada }(\mathrm{mg})\end{array}$ & $\begin{array}{c}\text { Massa de P } \\
\text { recuperada } \\
(\overline{\mathrm{X}} \mathrm{mg} \pm s)\end{array}$ & \% Recuperação \\
\hline Massa $(\mathrm{mg})$ & 0,57 & $0,49 \pm 0,04$ & $85,75 \pm 6,76$ \\
E09-SD01 & 1,14 & $0,97 \pm 0,01$ & $85,10 \pm 0,79$ \\
1000,0 & 1,71 & $1,76 \pm 0,02$ & $102,66 \pm 1,18$ \\
\hline$\overline{\bar{X}}$ - média das determinações, $s$ - estimativa do desvio padrão.
\end{tabular}

$\overline{\mathrm{X}}$ - média das determinações, $s$ - estimativa do desvio padrão.

A recuperação está relacionada com a exatidão, uma vez que compara a quantidade do analito recuperado no processo com a 
quantidade real presente na amostra. Os resultados revelaram que o método apresentou recuperações na faixa de 85,10-102,66\%, aceitáveis para o desenvolvimento de métodos analíticos.

\section{CONCLUSÕES}

O método desenvolvido para determinação do fósforo em paleossedimentos apresentou valores aceitáveis para as concentrações das amostras testadas, quando comparadas a um método tradicional. Os estudos sobre a massa, tempo, granulometria e spot permitiram ajustar os melhores parâmetros de trabalho, e os resultados dos ensaios de precisão e de adição e recuperação de analito apontaram o método como preciso e exato. Assim, ele pode ser usado na determinação do fósforo sedimentológico in situ, uma vez que a metodologia empregada é simples, rápida e não requer a etapa de pré-tratamento da amostra.

Ressalta-se que tais resultados são de importância fundamental ao trabalho arqueológico, seja na realização de busca de vestígios por meio de prospecção geoquímica ou para eleger os locais onde se deve efetuar as escavações em um sítio arqueológico, evitando assim perda de tempo.

\section{MATERIAL SUPLEMENTAR}

A Figura 1S apresenta o comportamento do sinal analítico do fósforo na linha $2,014 \mathrm{keV}$, obtido por p-ED-XRF, considerando o parâmetro granulometria, e a Figura $2 \mathrm{~S}$ mostra os estudos de interferencia espectral em determinações analíticas do fósforo utilizando o p-ED-XRF. Elas estão disponíveis em http://quimicanova. sbq.org.br, com acesso livre e em formato pdf.

\section{AGRADECIMENTOS}

Os autores são gratos às agências de fomento Conselho Nacional de Desenvolvimento Científico e Tecnológico (CNPq) e ao Fundo de Amparo à Pesquisa do Estado do Piauí (FAPEPI - PPP - 033/2019) pelo apoio financeiro, e à Fundação Museu do Homem Americano (FUMDHAM) pela liberação das amostras para estudo.

\section{REFERÊNCIAS}

1. Cavalcante, L. C. D.; Dissertação de Mestrado, Universidade Federal do Piauí, Brasil, 2008.

2. Hollyday, V. T.; Gartner, W. G.; J. Archaeol. Sci. 2007, 34, 301.

3. Marwick, B.; J. Archaeol. Sci. 2005, 32, 1357.

4. Moraes, B. C.; Moura, L. S.; Carvalho, R. C. P. S.; Corrêa, A. C. B.; Physis Terrae 2019, 1, 185.

5. Melo, C. R. S.; Farias Filho, B. B.; Anais do XXVII Seminário De Iniciação Científica - UFPI, Teresina, Brasil, 2018.

6. Villagran, X. S.; Schaefer, C. E. G. R.; Vestígios - Revista latinoamericana de Arqueologia Histórica 2011, 5, 117.

7. Miller, N. F. Em The Archaeology of Garden and Field; Miller, N. F., Gleason, K. L., eds.; University of Pennsylvania Press: Philadelphia, 1994, cap 2.

8. Provan, D. M. J.; Norw. Archaeol. Rev. 1971, 4, 37.

9. Lage, M. C. S. M.; Cavalcante, L. C. D.; Santos, J. S.; Fumdhamentos 2007, 6, 106.

10. Bicho, N. F.; Manual de Arqueologia Pré-Histórica. Lisboa: Portugal, 2006.
11. Santos, D. R.; Gatiboni, L. C.; Kaminski, J.; Cienc. Rural. 2008, 38, 576.

12. Barrow, N. J.; J. Soil Sci. 1983, 34, 733.

13. Eidt, R. C.; Science 1977, 197, 1327.

14. Parenti, F.; Fumdhamentos 1996, 1, 15.

15. Guidon, N.; Revista do Patrimônio Histórico e Artístico Nacional 2007, 33,75 .

16. Leite, L.; Cadernos do LEPAARQ (UFPEL) 2016, 13, 124.

17. Guidon, N.; Fumdhamentos 2008, 7, 380.

18. http://www.comciencia.br/dossies-1-72/reportagens/arqueologia/arq10. shtml, acessada em Agosto 2020.

19. Williams, J. D. H.; Syers, J. K.; Walker, T. W.; Soil Sci. Soc. Am. Proc. 1967, 31, 736.

20. Dick, W. A.; Tabatabai, M. A.; Soil Sci. Soc. Am. J. 1977, 41, 511.

21. Conway, J. S.; J. Archaeol. Sci. 1983, 10, 117.

22. Guttmann, E. B.; Simpson, I. A.; Davidson, D. A.; Dockrill, S. J.; Geoarchaeology 2006, 21, 61.

23. Simpson, I. A.; Guttmann, E. B.; Cluett, J. P.; Shepard, A.; Geoarchaeology 2006, 21, 221.

24. Linderholm, J.; Lundberg, E.; J. Archaeol. Sci. 1994, 21, 303.

25. Middleton, W. D.; Price, T. D.; J. Archaeol. Sci. 1996, 23, 673.

26. Murphy, J.; Riley, J. P.; Anal. Chim. Acta 1962, 27, 31.

27. Cavalcante, L. C. D.; Clio. Série Arqueológica (UFPE) 2009, 24, 119.

28. Cavalcante, L. C. D.; Lage, M. C. S. M.; Clio. Série Arqueológica (UFPE) 2010, 25, 11.

29. Torres, A. M.; Bastos, M. L. L.; Boaventura, G. R.; Licht, O. A. B.; Kern, D. C.; Melo Junior, G.; Frazão, F. J. L.; Costa, M. L.; Borba, R. P.; Figueredo, B. R. A.; Quartenário do Brasil 2005, 14, 298.

30. Ribeiro, B. T.; Silva, S. H. G.; Silva, E. A.; Guilherme. L. R. G.; Ciênc. Agrotec. 2017, 41, 245.

31. Hunt, A. M. W.; Speakman, R. J.; J. Archaeol. Sci. 2015, 53, 626.

32. Neff, H.; Bigney, S. J.; Sakai, S.; Burger, P. R.; Garfin, T.; George, R. G.; Culleton, B. J.; Kennett, D. J.; Appl. Spectrosc. 2016, 70, 110.

33. Gallhofer, D.; Lottermoser, B. G.; Minerals 2018, 8, 320.

34. Steiner, A.E.; Conrey, R. M.; Wolff, J. A.; Chem. Geol. 2017, 35, 453.

35. Neves, R. C. F.; Lima, P. M.; Baldassini, W. A. I.; Santos, F. A.; Moraes, P. M.; Castro, G. R.; Padilha, P. M.; Quim. Nova. 2012, 35, 493.

36. Padilla, J. T.; Hormes, J.; Magdi Selim, H.; Geoderma 2019, 337, 143.

37. Ravansari, R.; Wilson, S. C.; Tighe, M.; Environ. Int. 2020, 134, 105250.

38. Bugdalski, L.; Lemke, L. D.; Mc Elmurry, S. P.; Risk Analysis 2014, 34, 17.

39. Sutherland, R. A.; Environ. Pollut. (Oxford, U. K.) 2003, 121, 229.

40. https://www.epa.gov/sites/production/files/2015-12/documents/6200. pdf, acessada em Agosto 2020.

41. Rostron, P. D.; Ramsey, M. H.; Geostand. Geoanal. Res. 2017, 43, 459.

42. Kalnicky, D. J.; Singhvi, R.; J. Hazard. Mater. 2001, 83, 93

43. Parsons, C.; Margui Grabulosa, E.; Pili, E.; Floor, G. H.; Roman-Ross, G.; Charlet, L.; J. Hazard. Mater. 2013, 262, 1213.

44. Tighe, M.; Rogan, G.; Wilson, S. C.; Grave, P.; Kealhofer, L.; Yukongdi, P.; J. Environ. Manage. 2018, 206, 373.

45. Kilbride, C.; Poole, J.; Hutchings, T. R.; Environ. Pollut. (Oxford, U. K.) 2006, $143,16$.

46. Herreros-Chavez, L.; Cervera, M. L.; Morales-Rubio, A.; Food Chem. 2019, 278, 373 .

47. Brito, G. B.; Teixeira, L. S. G.; Korn, M. G. A.; Microchem. J. 2017, 134, 35 .

48. Costa, V. C.; Amorim, F. A. C.; Babos, D. V. Pereira-Filho, E. R.; Food Chem. 2019, 273, 91. 